\title{
A Novel Weighted-Counter Method for Simulating Coherent TWDP Fading Channels
}

\author{
Maria Luisa Merani, Senior Member, IEEE and Norman C. Beaulieu, Fellow, IEEE
}

\begin{abstract}
New channel simulation models are needed for the study of future wireless networks, 5G and beyond. A novel simulation technique dubbed weighted-counting is proposed. It is used to generate random variates having the two-wave with diffuse power (TWDP) fading amplitude distribution. The TWDP channel model describes high-speed train, femtocell, sea surface, heterogeneous and other channels having two different dominant line-of-sight waves as well as scatter power components.
\end{abstract}

Index Terms-Fading channels, line of sight, random variate generation, simulation, two-wave with diffuse power (TWDP), weighted-counter.

\section{INTRODUCTION}

$\mathbf{T}$ HE two-wave with diffuse power (TWDP) fading model is an important model for present and future wireless communication systems [1]- [7]. In this model, the received signal has two dominant specular components of comparable powers, plus numerous lower power scatter components of comparable powers. This model can represent high-speed train, femtocell, sea surface, heterogeneous and other channels, so statistically accurate TWDP fading simulation is needed. Yet, to the best of the authors' knowledge, no efficient method for generating TWDP amplitude random variates has been reported in the literature.

The contributions of this letter are first, to propose a novel simulation method dubbed, "weighted counting", and second to study how the weighted counting method can be used to simulate the amplitude of two-wave with diffuse power (TWDP) fading channels. In theory, the direct method [8] can be used to generate random variates having any probability distribution. However, in practice the direct method sometimes can not be used in cases where the inverse function of the cumulative probability distribution (CDF) is not known, or is not known in a simple form. While numerical inversion of the CDF can be efficiently employed in some cases, it may be complicated, or it may require extensive tables of coefficients for different parameter values that define the CDF [9]. The direct method sometimes cannot be used in cases where the inverse TWDP CDF function is not known. Numerical inversion of the CDF is not viable because the TWDP distribution has three degrees of freedom.

In the alternative, the rejection method of generating random variates is simple and effective in some cases where the direct

M. L. Merani is with DIEF, Dipartimento di Ingegneria Enzo Ferrari, University of Modena and Reggio Emilia, Modena 41121, Italy (e-mail: marialuisa.merani@unimore.it).

Norman C. Beaulieu is with the School of Information and Communication Engineering, and the Beijing Key Laboratory of Network System Architecture and Convergence, Beijing University of Posts and Telecommunications, Beijing100876, China (e-mail: nborm@bupt.edu.cn). method is not viable [10]. This method depends on finding a suitable overbounding PDF for the desired PDF. The efficiency of the method is determined by the selection of the overbounding PDF (also dubbed the "hat" function) and may be poor for some probability distributions. The rejection method was deemed not efficient for simulating TWDP channels, as we were unable to find a highly efficient hat function.

To the best of the authors' knowledge, weighted-counting simulation as proposed here has not previously been proposed in the literature. The remainder of this paper is organized as follows. The weighted-counter scheme is proposed in Section II and the TWDP fading simulator is designed in Section III. An application of the simulation of TWDP channels to receiver diversity is given in Section IV. Section V concludes the paper.

\section{A Weighted-Counter Scheme For Simulation}

We consider simulation estimates that are based on counting; this includes estimators of CDFs, PDFs, outage probability, and symbol error rates, for example. We are particularly concerned with the performance of wireless digital transmission systems subject to fading. Define the random fading $1 \times T$ row vector $\bar{R}=\left[R_{1}, R_{2}, \ldots, R_{T}\right]$, where the $R_{t}, t=1, \ldots, T$ are random variables representing fadings, and the random "nuisance" $1 \times M$ row vector $\bar{X}=\left[X_{1}, X_{2}, \ldots, X_{M}\right]$, where the random variables $X_{m}, m=1, \ldots, M$ may be, for example, Gaussian noises or cochannel interferences. Let $f_{\bar{R}}(\bar{r})$ be the joint probability density (PDF) of the random fading vector, $\bar{R}$, and $f_{\bar{X}}(\bar{x})$ be the joint PDF of the random nuisance vector, $\bar{X}$. In fading, some of the components of $\bar{X}$ may be functions of $\bar{R}$; for example, cochannel interferers may be subject to fading, so we define $f_{\bar{X} \mid \bar{R}}(\bar{x} \mid \bar{r})$ as the PDF of $\bar{X}$ conditioned on the fading, $f_{\bar{R}}(\bar{r})$. Generally, a performance measure of the system is given by the multiple integral expression ${ }^{1}$,

$$
p=\int_{0}^{\infty} \int_{0}^{\infty} \cdots \int_{0}^{\infty} f_{\bar{R}}(\bar{r}) \iint_{E} \ldots \int f_{\bar{X} \mid \bar{R}}(\bar{x} \mid \bar{r}) d \bar{x} d \bar{r}(1)
$$

where the hypervolume $E$ in $M$-dimensional hyperspace represents the region where the event occurs. Computer simulation is widely used to estimate the integral in (1) because of the complexity and stability issues related to computing nested integrals, and because the amount of numerical computations involved grows exponentially with the number of levels of inte-

\footnotetext{
${ }^{1}$ In the model, we permit $f_{\bar{X} \mid \bar{R}}(\bar{x})=\delta\left(x_{1}\right) \delta\left(x_{2}\right) \cdots \delta\left(x_{M}\right)$, so that $\bar{X}=$ $\overline{0}$. Then the model includes outage probability as an event, for which the multiple integrations over $\bar{X}$ equals unity.
} 
gration. Let $\widehat{p}$ denote the estimate of $p$ obtained by simulation, which is given by

$$
\widehat{p}=\frac{\sum_{j=1}^{N_{f}} \sum_{i=1}^{N} I\left(\bar{r}_{j}, \bar{x}_{i}\right)}{N \times N_{f}}
$$

the indicator function $I\left(\bar{r}_{j}, \bar{x}_{i}\right)$ taking on the unitary value when the event occurs, and the value zero when it does not. $N$ and $N_{f}$ are the number of nuisance vectors and fading vectors, respectively, generated in the simulation, and where $\bar{r}_{j}$ and $\bar{x}_{i}$ denote the $j$ th vector of fading random variates and the $i$ th vector of nuisance random variates, respectively. The joint PDFs, $f_{\bar{X}_{i} \mid \bar{R}_{j}}\left(\bar{x}_{i} \mid \bar{r}_{j}\right)$ and $g_{\bar{R}_{i}}\left(\bar{r}_{i}\right)$, of the random vectors $\bar{X}_{i}$ and $\bar{R}_{j}$ representing the $i$ th nuisance vector and $j$ th fading vector, respectively, of the simulation do not depend on $i$ and $j$, respectively and have common PDFs $f_{\bar{X} \mid \bar{R}}(\bar{x} \mid \bar{r})$ and $g_{\bar{R}}(\bar{r})$, respectively. The estimator $\widehat{p}$ in (2) is an unbiased estimator of $p$, as its mean equals $p$, i.e.,

$$
\begin{aligned}
& E[\hat{p}]=\frac{N \cdot N_{f} \times E\left[I\left(\bar{r}_{j}, \bar{x}_{i}\right)\right]}{N \cdot N_{f}} \\
& =\int_{0}^{\infty} \int_{0}^{\infty} \cdots \int_{0}^{\infty} f_{\bar{R}}(\bar{r}) \int_{-\infty}^{\infty} \int_{-\infty}^{\infty} \cdots \int_{-\infty}^{\infty} f_{\bar{X} \mid \bar{R}}(\bar{x} \mid \bar{r}) \\
& \left.=\int_{0}^{\infty} \int_{0}^{\infty} \cdots \int_{0}^{\infty} f_{\bar{R}}(\bar{r}) \iint_{E} \ldots \int f_{\bar{X} \mid \bar{R}}(\bar{x}) d \bar{x} d \bar{r}\right) d \bar{x} d \bar{r}=p
\end{aligned}
$$

since the indicator function $I(\bar{r}, \bar{x})$ is 1 inside the region $E$, and 0 outside. Now consider the case when the evaluation of estimate $\widehat{p}$ is rather cumbersome, because the generation of the samples of the $\bar{R}$ vector is complex; this is, for example, the case for the TWDP fading channel. What we observe is that, in lieu of estimating $\widehat{p}$ by (2), we can resort to

$$
\hat{p}_{w c}=\frac{\sum_{j=1}^{N_{f}} W\left(\bar{r}_{j}\right) \sum_{i=1}^{N} I\left(\bar{r}_{j}, \bar{x}_{i}\right)}{N \times N_{f}}
$$

where the statistical description of the $\bar{r}_{j}$ components has been replaced by an alternative, more tractable joint PDF, $g_{\bar{R}}(\bar{r})$, subject to the constraint $g_{\bar{R}}(\bar{r}) \neq 0$ where $f_{\bar{R}}(\bar{r}) \neq 0$, and the $W\left(\bar{r}_{j}\right), j=1,2, \ldots, N_{f}$ represent proper weights,

$$
W\left(\bar{r}_{j}\right)=\frac{f_{\bar{R}}\left(\bar{r}_{j}\right)}{g_{\bar{R}}\left(\bar{r}_{j}\right)} .
$$

It must be verified that $\hat{p}_{w c}$ is again an unbiased estimator of $p$. Taking the average of (6) and making use of (7) gives,

$$
\begin{aligned}
& E\left[\widehat{p}_{w c}\right]=\frac{N \cdot N_{f} \times E[W(\bar{r}) \times I(\bar{r}, \bar{x})]}{N \cdot N_{f}} \\
& =\int_{0}^{\infty} \ldots \int_{0}^{\infty} \frac{f_{\bar{R}}(\bar{r})}{g_{\bar{R}}(\bar{r})} \cdot\left\{\int_{-\infty}^{\infty} \ldots \int_{-\infty}^{\infty} I(\bar{r}, \bar{x})\right. \\
& \left.=\int_{0}^{\infty} \int_{0}^{\infty} \ldots \int_{0}^{\infty} f_{\bar{R}}(\bar{r}) \iint_{\bar{X} \mid \bar{R}}(\bar{x} \mid \bar{r}) d \bar{x}\right\} g_{\bar{R}}(\bar{r}) d \bar{r} \\
& f_{\bar{X} \mid \bar{R}}(\bar{x} \mid \bar{r}) d \bar{x} d \bar{r}=p .
\end{aligned}
$$

In the sequel, it will be convenient to consider the case where the $T$ random components of $\bar{R}_{j}$ are mutually independent, so that one has

$$
\begin{gathered}
W\left(\bar{r}_{j}\right)=\frac{f_{R_{j 1}}\left(r_{j 1}\right)}{g_{R_{j 1}}\left(r_{j 1}\right)} \cdot \frac{f_{R_{j 2}}\left(r_{j 2}\right)}{g_{R_{j 2}}\left(r_{j 2}\right)} \cdots \frac{f_{R_{j T}}\left(r_{j T}\right)}{g_{R_{j T}}\left(r_{j T}\right)} \\
=W\left(r_{j 1}\right) \cdot W\left(r_{j 2}\right) \cdots W\left(r_{j T}\right)
\end{gathered}
$$

where we define $W\left(r_{j t}\right)$ as the marginal weight associated with the $t$ th random variable of the $j$ th vector of random variables, and where $f_{R_{j t}}\left(r_{j t}\right)$ and $g_{R_{j t}}\left(r_{j t}\right)$ for $t=1,2, \ldots, T$ are, respectively, the marginal PDFs of the multivariate PDFs $f_{\bar{R}}\left(\bar{r}_{j}\right)$ and $g_{\bar{R}}\left(\bar{r}_{j}\right)$.

\section{GENERATING RANDOM VARIATES FROM TWDP CHANNELS}

We design a weighted-counter scheme to simulate the amplitude of TWDP fading channels, that can be used to simulate outage probabilities and other performance metrics such as symbol error rate in systems employing coherent modulation schemes assumed to have phase recovery. The expressions for the PDF of the random amplitude, $R$, of TWDP fading derived in [1, eq. (29)] and [3, eq. (2)] are in integral forms, namely

$$
f_{R}(r)=r \int_{0}^{\infty} e^{-\frac{\sigma^{2} v^{2}}{2}} J_{0}(r v) J_{0}\left(V_{1} v\right) J_{0}\left(V_{2} v\right) v d v
$$

and

$$
\begin{aligned}
f_{R}(r)= & \frac{r}{\sigma^{2}} e^{-\frac{r^{2}}{2 \sigma^{2}}-K} \\
& \times \frac{1}{\pi} \int_{0}^{\pi} e^{K \Delta \cos \theta} I_{0}\left(\frac{r}{\sigma} \sqrt{2 K(1-\Delta \cos \theta)}\right) d \theta
\end{aligned}
$$

respectively, where $2 \sigma^{2}$ is the power of the scatter components, $V_{1}$ and $V_{2}$ are the amplitudes of the two specular components, $J_{0}(\cdot)$ is the regular Bessel function of the first kind of order zero, $K$ is a distribution shape parameter equal to the ratio of the total power in the two line-of-sight components to the power in the scatter components (similar to the Ricean $K$ factor), and $I_{0}(\cdot)$ is the modified Bessel function of the first kind of order zero. The parameter $\Delta$ is also a shape parameter taking values between 0 and 1 , defined to relate the relative magnitudes of $V_{1}$ and $V_{2}$ to one another [10]. More detailed explanations and intuitive meanings of the parameters are given in Ref. [1] and cannot be repeated here due to space limitations. The TWDP PDF is very complicated, and it is not feasible to apply the direct method to eq. (11). Therefore, we design a simulator for TWDP fading channels by inventing the weighted-counter scheme.

The weighted-counter technique is most effective when $g_{\bar{R}}(\bar{r})$ is "close" to $f_{\bar{R}}(\bar{r})$ so that the weights do not become too large or too small. In this regard, one should pay attention to the ratio of the values of the tails of $g_{\bar{R}}(\bar{r})$ and $f_{\bar{R}}(\bar{r})$ for large arguments. Here, we choose $g_{R_{j t}}\left(r_{j t}\right), t=1, \ldots, T$ as the PDF for the Ricean channel, and $f_{R_{j t}}\left(r_{j t}\right), t=1, \ldots, T$ 
as the PDF for the TWDP channel, so that,

$$
\begin{aligned}
& g_{R_{j t}}\left(r_{j t}\right)=\frac{r_{j t}}{\sigma^{2}} e^{-\frac{r_{j t}^{2}}{2 \sigma^{2}}-K} I_{0}\left(\frac{r_{j t}}{\sigma} \sqrt{2 K}\right) \\
f_{R_{j t}}\left(r_{j t}\right)= & \frac{r_{j t}}{\sigma^{2}} e^{-\frac{r_{j t}^{2}}{2 \sigma^{2}}-K} \\
& \times \frac{1}{\pi} \int_{0}^{\pi} e^{K \Delta \cos \theta} I_{0}\left(\frac{r_{j t}}{\sigma} \sqrt{2 K(1-\Delta \cos \theta)}\right) d \theta .
\end{aligned}
$$

Then, the counter weight $W\left(\bar{r}_{j}\right)$ becomes

$$
\begin{aligned}
W\left(\bar{r}_{j}\right) & = \begin{cases}\frac{f_{\bar{R}}\left(\bar{r}_{j}\right)}{g_{\bar{R}}\left(\bar{r}_{j}\right)}, & \text { if the event occurs } \\
0, & \text { if the event does not occur }\end{cases} \\
& =\left\{\begin{array}{cc}
\prod_{t=1}^{T} f_{R_{j t}}\left(r_{j t}\right) & \text { if the event occurs } \\
\prod_{t=1}^{T} g_{R_{j t}}\left(r_{j t}\right) & \text { if the event does not occur } \\
0, & \text { if the event occurs }
\end{array}\right. \\
& =\left\{\begin{array}{cc}
{\left[W\left(r_{j t}\right)\right]^{T},} & \text { if the event does not occur } \\
0, &
\end{array}\right.
\end{aligned}
$$

where

$$
\begin{aligned}
W\left(r_{j t}\right) & =\frac{f_{R_{j t}}\left(r_{j t}\right)}{g_{R_{j t}}\left(r_{j t}\right)} \\
& =\frac{\int_{0}^{\pi} e^{K \Delta \cos \theta} I_{0}\left(\frac{r_{j t}}{\sigma} \sqrt{2 K(1-\Delta \cos \theta)}\right) d \theta}{\pi I_{0}\left(\frac{r_{j t}}{\sigma} \sqrt{2 K}\right)}
\end{aligned}
$$

because the $r_{j t}$ are independent and identically distributed.

The value of the weights might seem to be an important issue; perhaps very large or small weights compared to the value 1 could result in inaccurate computations. To illustrate, imagine that the weights need to assume values greater than $10^{20}$ or less than $10^{-20}$, while some of the weights have values close to 1 . Then, some of the events go lost in the counting process because of finite numerical precision in the computations. Note that the dynamic range of the weights is determined by the choice of the functions $g(r)$ and $f(r)$.

To study this question further, consider the relative magnitudes of the weights for TWDP fading. The weights are functions of the parameters $\frac{r_{j t}}{\sigma}, K$, and $\Delta$; that is,

$$
W\left(r_{j t}\right)=W\left(\frac{r_{j t}}{\sigma} ; K, \Delta\right) .
$$

Fig. 1 shows the weight, $W\left(\frac{r_{j t}}{\sigma} ; K, \Delta\right)$, as a function of $\frac{r_{j t}}{\sigma}$ for $K=6 \mathrm{~dB}$ and four different values of $\Delta$. When $\Delta=0$, the TWDP fading distribution becomes the Ricean fading distribution and the weight $W\left(\frac{r_{j t}}{\sigma} ; K, 0\right)=1$, as seen in Fig. 1. Observe that the counter weight becomes large for large values of $\frac{r_{j t}}{\sigma}$ and for $\frac{r_{j t}}{\sigma}=0$. It also increases as $\Delta$ ranges from 0 to 1 . Note that values of the weight function greater than 1 must infer that there are also values of the weight function less than 1. Fig. 1 shows, therefore, regions where $W\left(\frac{r_{j t}}{\sigma} ; K, \Delta\right)>1$ and regions where $W\left(\frac{r_{j t}}{\sigma} ; K, \Delta\right)<1$. We have found empirically that there are no problems in simulating values of $K \leq 12 \mathrm{~dB}$ (the simulations were implemented on the Matlab simulator in the Windows operating system). Note that for values of $K>12 \mathrm{~dB}$, the probabilities involved are so small as to facilitate asymptotic analysis or as to require special simulation techniques.

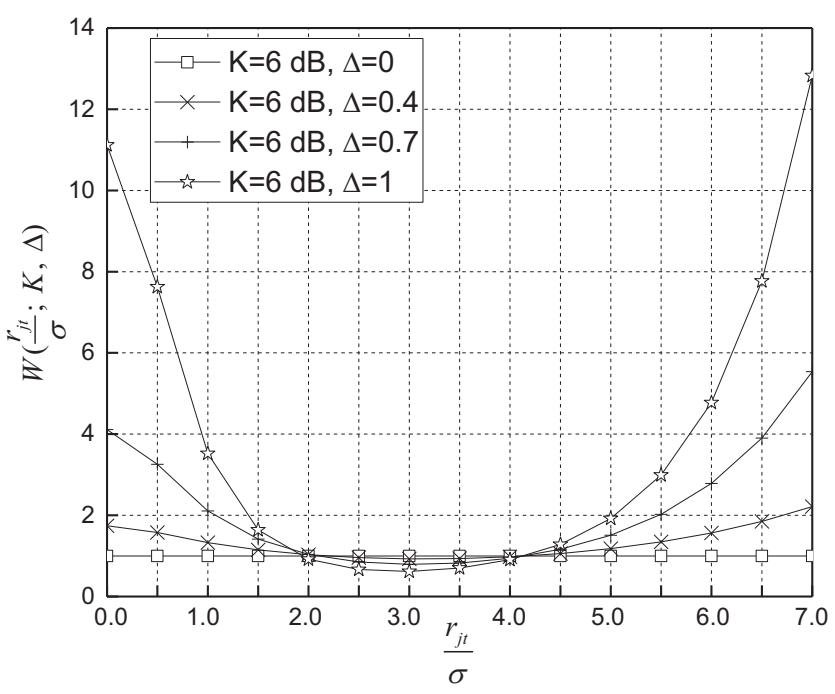

Fig. 1. The weight $W\left(\frac{r_{j t}}{\sigma} ; K, \Delta\right)$ as a function of $\frac{r_{j t}}{\sigma}$ for $K=6 \mathrm{~dB}$ and four different values of $\Delta$, with fixed scatter component power ( $\sigma^{2}$ fixed).

Theoretical PDF and CDF curves are shown in Fig. 2 and Fig. 3, respectively, and simulated PDF and CDF curves obtained using the weighted-counter method are also shown. Excellent agreement between theoretical and simulation results is seen in all cases.

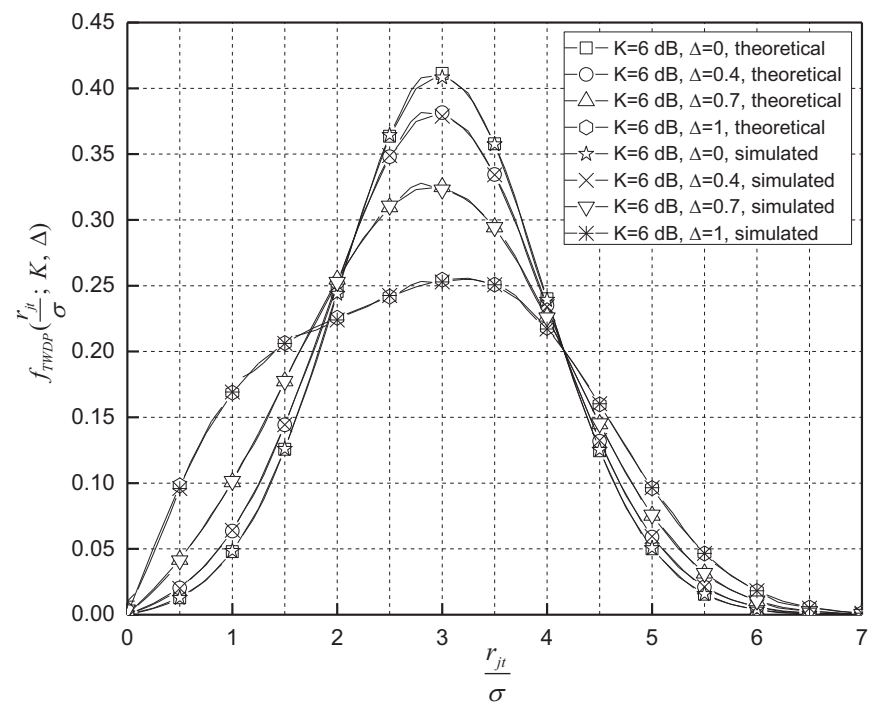

Fig. 2. The theoretical and simulated PDFs of TWDP fading amplitude for $K=6 \mathrm{~dB}$ and four different values of $\Delta$

\section{APPLICATION}

As an example of the weighted-counter simulation scheme, we study the outage probability of equal gain combining (EGC) diversity [11], [12] in a three branch diversity system. EGC diversity is a coherent scheme and so only the amplitude of the faded signal needs to be simulated to determine, outage, 


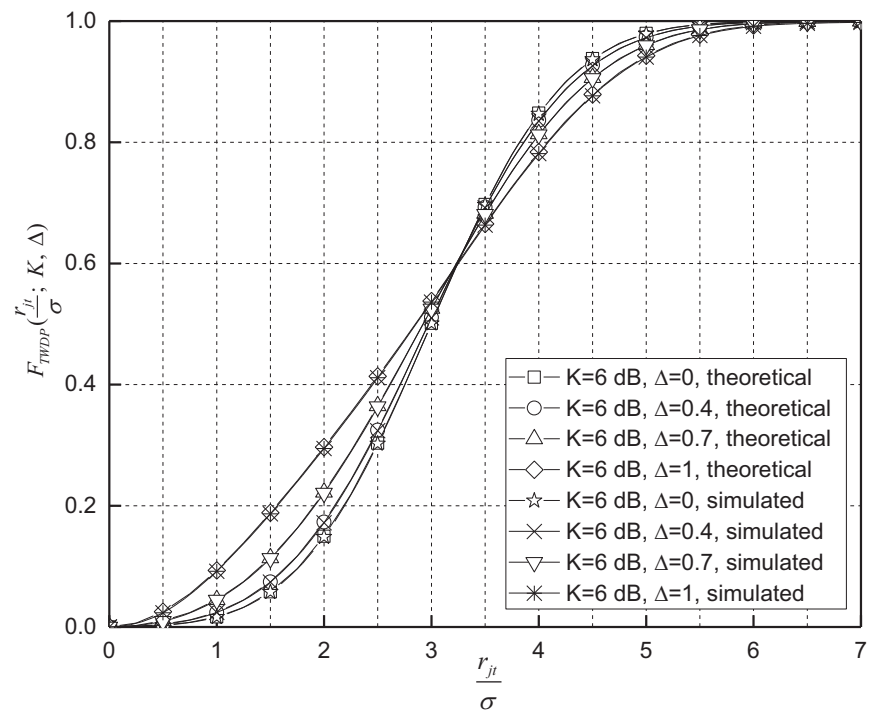

Fig. 3. The theoretical and simulated CDFs of TWDP fading amplitude for $K=6 \mathrm{~dB}$ and four different values of $\Delta$, with fixed scatter component power ( $\sigma^{2}$ fixed).

symbol error probability, packet rate, packet interarrival time statistics, etc. Note that there are no closed-form solutions for these problems, and that the problem of finding a closed-form solution for the PDF of EGC for 3 or more diversity branches is extremely difficult (see references [11] and [13] and references therein). Furthermore, the characteristic function of the TWDP distribution is not known in tractable closed-form. These facts preclude analytical solutions to these problems, so weighted-counter simulation is a good choice for tractable performance evaluation.

Fig. 4 shows the outage probability of EGC operating on a TWDP channel with $K=6 \mathrm{~dB}$ for four different values of $\Delta$. At $10 \mathrm{~dB}$, when the TWDP parameters reflect a poor channel $(\Delta=1), 3$-fold EGC diversity improves the outage probability from about $10^{-1}$ to $3 \times 10^{-4}$, making an unusable channel usable. On a better TWDP channel $(\Delta=0.4), 3$-fold EGC diversity improves the outage probability from about $10^{-1}$ to $3 \times 10^{-4}$ at $5 \mathrm{~dB}$. When $\Delta=0$, the TWDP channel becomes a Ricean channel, which is seen to perform best at high SNR in this example. Interestingly, as seen by careful inspection of the curves at small values of SNR (less than $-4.8 \mathrm{~dB}$ ), the ordering of the channels from best to worst is reversed from the ordering at moderate to large SNR.

\section{CONCLUSION}

Refined new channel models enable improved utilization of wireless channels, but may entail complicated amplitude distribution functions which cannot be usefully inverted. The direct method cannot be used to generate random variates for simulation when the inverse CDF function is not known, and rejection methods may suffer poor efficiencies. A novel weighted-counting simulation technique was proposed for such cases. As an example, the method was applied to obtain new results for the outage probabilities of 3-branch EGC on TWDP channels for different choices of the TWDP channel

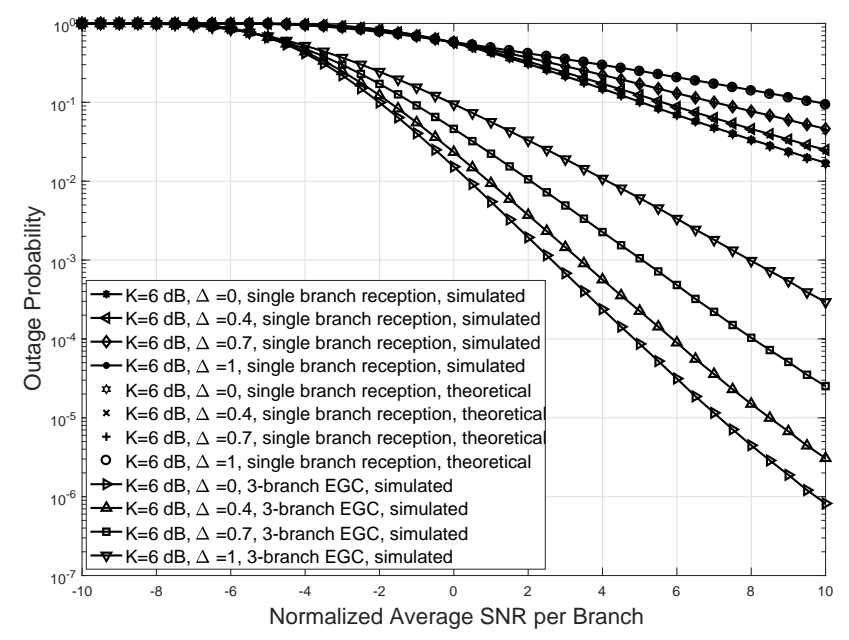

Fig. 4. Comparison of the outage probability of 3-branch EGC and single branch reception operating on a TWDP channel for $K=6 \mathrm{~dB}$ and four different values of $\Delta$. The normalized average SNR $=\frac{\bar{\gamma}}{\gamma_{t h r}}$, where $\bar{\gamma}$ and $\gamma_{t h r}$ are average SNR and outage threshold, respectively.

parameters. The weighted-counter simulation scheme will find application to other fading channel models whose CDFs are complex and/or not invertible.

\section{REFERENCES}

[1] G. D. Durgin, T. S. Rappaport, and D. A. de Wolf, "New analytical models and probability density functions for fading in wireless communications," IEEE Trans. Commun., vol. 50, no. 6, pp. 1005-1015, Jun. 2002.

[2] S. A. Saberali and N. C. Beaulieu, "New expressions for TWDP fading statistics," IEEE Wireless Commun. Lett., vol. 2, no. 6, pp. 643-646, Dec. 2013.

[3] M. Rao, F. Lopez-Martinez, M.-S. Alouini, and A. Goldsmith, "MGF approach to the analysis of generalized two-ray fading models," IEEE Trans. Wireless Commun., vol. 14, no. 5, pp. 2548-2561, May 2015.

[4] D. Dixit and P. Sahu, "Performance of QAM signaling over TWDP fading channels," IEEE Trans. Wireless Commun., vol. 12, no. 4, pp. 1794-1799, Apr. 2013.

[5] Y. Lu, X. Wang, and N. Yang, "Outage probability of cooperative relay networks in two-wave with diffuse power fading channels," IEEE Trans. Commun., vol. 60, no. 1, pp. 42-47, Jan. 2012.

[6] B. S. Tan, K. H. Li, and K. C. Teh, "Symbol-error rate of selection combining over two-wave with diffuse power fading," in IEEE 5th Int. Conf. Signal Processing and Communication Systems (ICSPCS), Dec. 2011, pp. $1-5$.

[7] S. Oh, K. Li, and W. Lee, "Performance of bpsk pre-detection mrc systems over two-wave with diffuse power fading channels," IEEE Trans. Wireless Commun., vol. 6, no. 8, pp. 2772-2775, Aug. 2007.

[8] A. Papoulis and S. U. Pillai, Probability, random variables, and stochastic processes, 4th ed. New York: McGraw-Hill, 2002.

[9] N. C. Beaulieu and C. Cheng, "Efficient Nakagami- $m$ fading channel simulation," IEEE Trans. Veh. Technol., vol. 54, no. 2, pp. 413-424, Mar. 2005.

[10] L. Cao and N. C. Beaulieu, "Simple efficient methods for generating independent and bivariate Nakagami- $m$ fading envelope samples," IEEE Trans. Veh. Technol., vol. 56, no. 4, pp. 1573-1579, Jul. 2007.

[11] N. C. Beaulieu and A. A. Abu-Dayya, "Analysis of equal gain diversity on Nakagami fading channels," IEEE Trans. Commun., vol. 39, no. 2, pp. 225-234, Feb. 1991

[12] D. G. Brennan, "Linear diversity combining techniques," IEEE Proc., vol. 91, no. 2, pp. 331-356, Feb. 2003.

[13] N. C. Beaulieu, "An infinite series for the computation of the complementary probability distribution function of a sum of independent random variables and its application to the sum of Rayleigh random variables," IEEE Trans. Commun., vol. 38, pp. 1463-1474, Sept. 1990. 